M.D. Rugg $\cdot$ P. Walla $\cdot$ A.M. Schloerscheidt

P.C. Fletcher - C.D. Frith - R.J. Dolan

\title{
Neural correlates of depth of processing effects on recollection: evidence from brain potentials and positron emission tomography
}

\begin{abstract}
The probability that words would be recollected during tests of recognition memory was varied by manipulating depth of processing at study. Experiment $1 \mathrm{em}-$ ployed scalp-recorded event-related potentials (ERPs), and identified as a correlate of recollection a late (onset c. $500 \mathrm{~ms}$ ), strongly left-lateralized positive-going modulation of the ERP waveform. The findings from experiment 2, which employed positron emission tomography (PET), indicated that recollection was associated with activation of the left hippocampal formation together with an extensive region of left temporal and frontal cortex. The findings support current ideas about the role of the hippocampal formation in episodic memory retrieval, and provide complementary information about the time course and localization of the cortical correlates of the recollection of recently experienced words.
\end{abstract}

Key words Event-related potentials - Hippocampus · Positron emission tomography $\cdot$ Recognition memory • Retrieval

\section{Introduction}

Memory for prior events can be fractionated in a number of ways (Rugg 1995a). Memories can be retrieved intentionally or incidentally; they can be accompanied or unaccompanied by awareness (explicit vs implicit memory); and explicit memory can be fractionated further into recollection, when the retrieved information includes contextual information about the learning episode and is ac-

M.D. Rugg ( $)$ A.M. Schloerscheidt

Wellcome Brain Research Group, School of Psychology,

University of St Andrews, St Andrews KY16 9JU, UK

e-mail: mdr@st-andrews.ac.uk, Fax: +44-1334-463 042

P. Walla

Department of Neurology, University of Vienna, Vienna, Austria

P.C. Fletcher - C.D. Frith · R.J. Dolan

Wellcome Department of Cognitive Neurology,

Institute of Neurology, University College London, London, UK companied by the phenomenological experience of "remembering", and familiarity, which is the feeling that an item has recently been experienced in the absence of any contextual information about the experience.

The two experiments described here employed scalprecorded event-related potentials (ERPs) and positron emission tomography (PET) respectively to obtain information about the neural correlates of the recollection of recently experienced words. The information provided by the two methods is highly complementary. ERPs are well suited to investigating the time course and functional properties of neural activity supporting cognition (Rugg and Coles 1995). But because of their poor spatial resolution, the identification of the neural structures responsible for such activity necessitates the employment of a neuroimaging method such as PET, which, although it provides little or no temporal information, is capable of localizing task-related changes in cerebral activity with a high degree of accuracy $(<1 \mathrm{~cm})$.

Neuropsychological evidence (Squire et al. 1993) indicates that the recollection of recently experienced events depends critically upon the integrity of the hippocampus and adjacent regions of the medial temporal cortex (henceforth, the hippocampal formation). Little is known, however, about how the hippocampal formation interacts with other brain regions, notably the neocortex, to support the retrieval and representation of episodic memories. According to some models of hippocampal function (e.g. McClelland et al. 1995), the role of this structure is to store information about the patterns of cortical activation that represented events at the time they were initially experienced, allowing these patterns to be reinstated subsequently in response to appropriate retrieval cues. According to such models, retrieval-related hippocampal activity should invariably be associated with modulation of cortical activity, this latter activity reflecting the reinstatement of the representation of the retrieved episode.

The two experiments described here take as their starting point the claim (Gardiner et al. 1996; but see Toth 1996) that the well-known effects of "depth" of study processing on subsequent retrieval (Craik and Lockhart 
1972) are mediated largely through the influence that this variable exerts on recollection rather than familiarity (or implicit memory). In both experiments the neural correlates of recollection were identified by contrasting the cerebral activity associated with the recognition of words studied either "deeply" or "shallowly". In the ERP study, neural activity linked to familiarity and implicit memory was also investigated, by looking for differences in the ERPs elicited by old and new words that were insensitive to variations in depth of study processing.

\section{Experiment 1}

Methods

ERPs were collected from the test phases of two study-test blocks. In each block subjects $(n=16)$ first studied a series of 68 visually presented words (plus 4 filler items). Each word was preceded by one of two fixation characters, which signalled which of two study tasks was to be performed with the upcoming word. In the deep task subjects were required to incorporate the word into a short spoken sentence. In the shallow task subjects judged whether the first and last letters of the word were in alphabetical order. After a break of approximately $5 \mathrm{~min}$ the test task was administered. This comprised a series of 102 critical words and 10 fillers, each presented for a duration of $300 \mathrm{~ms}$ with a stimulus onset asychrony of $5.3 \mathrm{~s}$. The critical words consisted of a random sequence of 34 unstudied (new) items and 68 studied (old) items; half of the latter had been subjected to deep encoding and half to shallow encoding. Subjects were required to classify each word as "old" or "new" by pressing one of two microswitch keys. Across subjects, words were rotated across categories (new, shallow, deep) to eliminate item effects.

Fig. 1 Experiment 1: grand average event-related potential (ERP) waveforms elicited by correctly classified new, shallowly studied (shallow hit) and deeply studied (deep hit) words. Waveforms are shown from electrode sites on the midline $(F z, C z$ and $P z$ ), and over left and right frontal $(L F, R F)$, anterior temporal $(L T, R T)$, parietal $(L P, R P)$, posterior temporal $(T 5, T 6)$, and occipital $(O 1, O 2)$ regions of the scalp
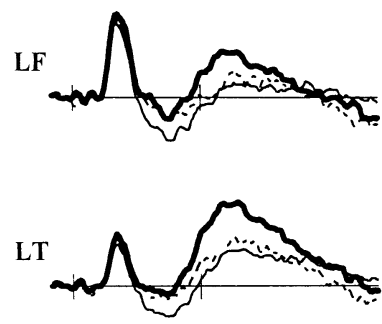

During the test tasks the electroencephalogram (EEG) was recorded [bandpass $(3 \mathrm{~dB}$ points] $0.03-30 \mathrm{~Hz})$ from 26 sites [Fz, $\mathrm{Cz}, \mathrm{Pz}, \mathrm{Fp} 1 / \mathrm{Fp} 2, \mathrm{~F} 7 / \mathrm{F} 8, \mathrm{LF} / \mathrm{RF}$ (75\% of the distance from $\mathrm{Fz}$ to F7/F8), F3/F4, T3/T4, LT/RT (75\% of the distance from $\mathrm{Cz}$ to T3/T4), C3/C4, T5/T6, LP/RP (75\% of the distance from $\mathrm{Pz}$ to $\mathrm{T} 5 / \mathrm{T} 6), \mathrm{P} 3 / \mathrm{P} 4, \mathrm{O} 1 / \mathrm{O} 2$, and right mastoid] with respect to a left mastoid electrode, and subsequently re-referenced to represent recordings with respect to linked mastoids. The electro-oculogram (EOG) was monitored by a bipolar electrode pair. ERPs (sampling rate $6 \mathrm{~ms} /$ point, $102 \mathrm{~ms}$ pre-stimulus baseline, post-stimulus epoch $1434 \mathrm{~ms}$ ) elicited by correctly classified new and old words were formed from all trials free from EOG or other artefact.

Results

\section{Behaviour}

Subjects correctly classified $86 \%$ of the new words, $94 \%$ of the deeply studied old words and $49 \%$ of the shallowly studied old words. The two hit rates were significantly different $(t 15=16.89, P<0.001)$.

\section{ERPs}

Grand average waveforms are shown for selected electrode sites in Fig. 1. From approximately 300 to $500 \mathrm{~ms}$ following stimulus onset, both classes of old word elicited ERPs that were more positive-going than those to new words (ANOVA of mean amplitude of the 300-500 ms latency region for the sites illustrated in Fig. 1: main effect
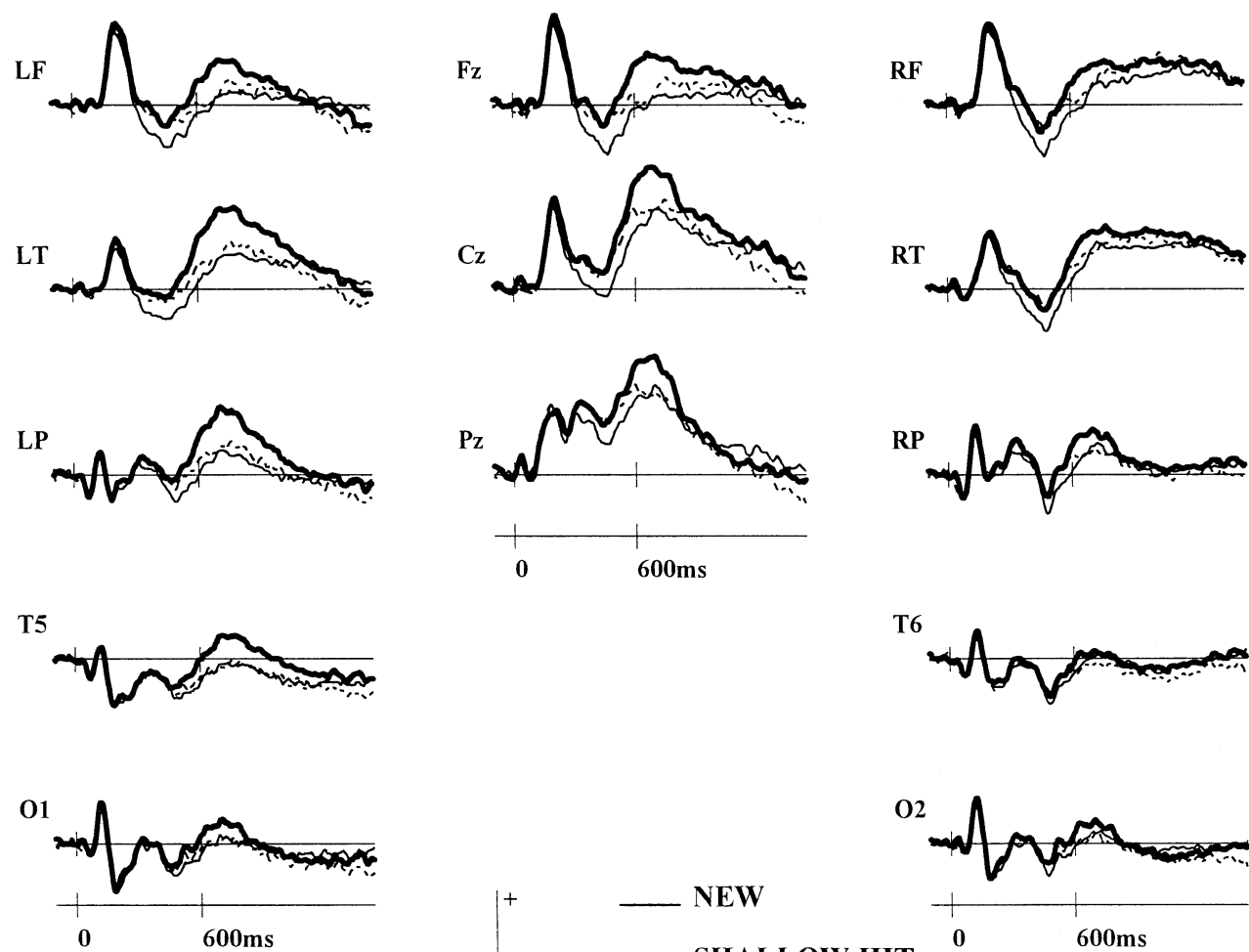

T6

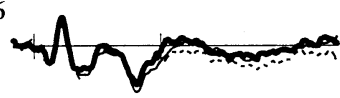

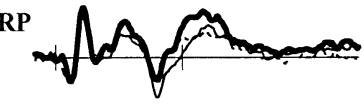

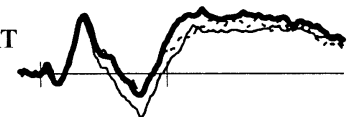

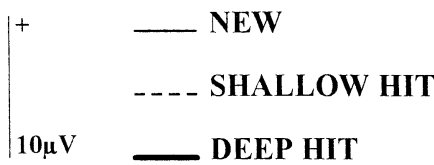

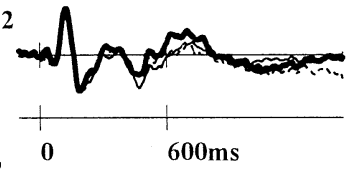




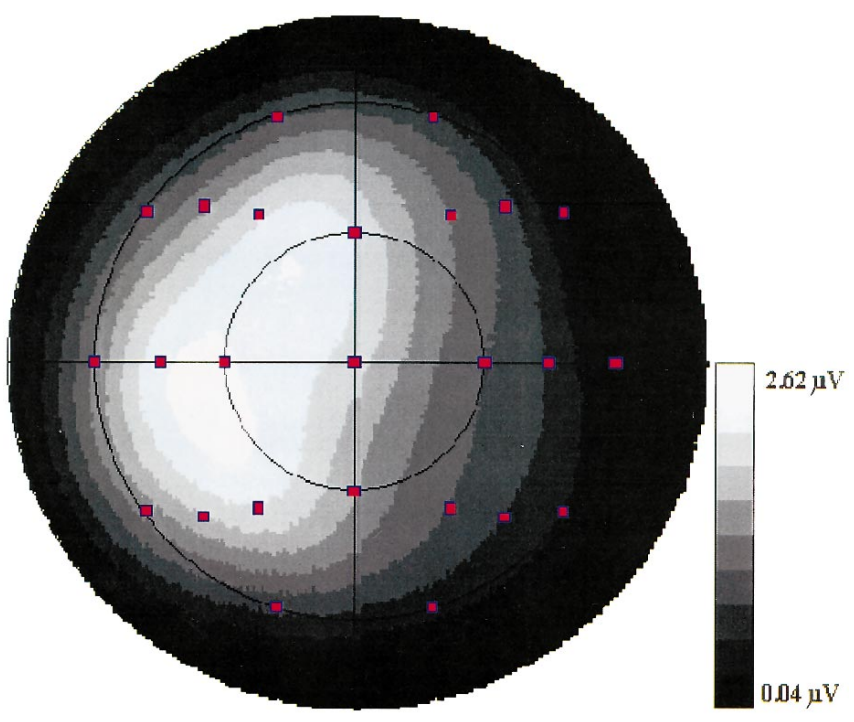

Fig. 2 Experiment 1: spherical spline map illustrating the scalp distribution of the voltage differences in the 500-800 ms latency region of the ERPs elicited by correctly classified deeply and shallowly studied words

of word class $\left.F_{2,29}=9.15, P<0.001\right)$. The ERPs elicited by the two classes of old word did not differ from one another $(F<1)$. From approximately 500 to $800 \mathrm{~ms}$, however, the ERPs to deeply studied old words were more positive-going than those elicited either by new items (mean amplitude 500-800 ms: main effect of word class $\left.F_{1,15}=30.20, P<0.001\right)$ or shallowly studied old words (mean amplitude 500-800 ms: main effect of word class $\left.F_{1,15}=14.15, P<0.005\right)$. As is evident from the statistical analyses and Fig. 1, these effects are markedly asymmetric, predominating over the left hemisphere. As might be expected from Fig. 1, the scalp topographies of the differences between the ERPs to the deeply studied and new words differed according to latency region (latency region $\times$ site interaction: $F_{4,52}=3.26, P<0.025$ ). The scalp topography of the differences between the ERPs elicited by the two categories of old item is illustrated in Fig. 2. In keeping with the impression given by this figure, ANOVA of the ERPs to these items from the lateral electrode sites illustrated in Fig. 1 revealed a reliable interaction between word class and hemisphere for the 500$800 \mathrm{~ms}$ latency region $\left(F_{1,15}=18.29, P<0.001\right)$, indicating that the word class differences were greater over the left than the right hemisphere.

\section{Discussion}

The behavioural findings indicate that the study manipulation was successful: considerably fewer words were recognized from the shallow study task than from the deep task. As already noted, such depth of processing effects are thought to arise mainly through their influence on recollection, which is highly sensitive to the degree of semantic processing and elaboration accorded items at the time of encoding. On this basis, we assume that the majority of the correctly classified old words subjected to deep encoding were recollected, whereas shallowly studied words engendered little recollection, and were recognized as old largely or solely on the basis of their familiarity.

The ERPs revealed two major findings. First, there was a region of the ERP waveform, between approximately 300 and $500 \mathrm{~ms}$ after stimulus onset, which differed according to whether items were old or new but was insensitive to the depth of processing manipulation. The lack of sensitivity of this effect to depth of processing suggests that it reflects neural activity associated with a form or forms of memory distinct from recollection. The two most obvious candidates are familiarity and implicit memory (see Introduction and Rugg et al. (1998).

The second noteworthy aspect of the ERP findings concerns the memory effect that occurred from approximately 500 to $800 \mathrm{~ms}$ after the stimulus. This effect differed from that noted above both in terms of its functional characteristics (it was highly sensitive to the depth of processing manipulation) and its scalp topography (which, unlike the earlier effect, was strongly left-lateralized).

The sensitivity of the post-500 ms memory effect to depth of processing is consistent with the proposal that it reflects neural activity associated with the recollection of test items' study episodes. In its time course and scalp topography, this effect closely resembles an ERP effect the "left parietal old/new effect" - that has been identified previously as an ERP correlate of recollection in studies of source memory (Wilding and Rugg 1996; see also Allan et al. 1998). It is likely that the neural population generating the present effect strongly overlaps with that responsible for the left parietal effect described in earlier studies. Thus, the present findings add weight to earlier claims (Paller and Kutas 1992; Smith 1993; Wilding and Rugg 1996; Allan et al. 1998) that this effect is an electrophysiological correlate of recollection.

Where are the generators of this recollection-sensitive effect? As noted in the Introduction, the ability to recollect recent events depends critically on the integrity of the hippocampal formation, leading to the expectation that this structure would be differentially active during the processing of items that elicit successful and unsuccessful recollection. While the neuropsychological evidence may appear to make the hippocampal formation a candidate generator of the ERP correlates of recollection described here and in previous work, it is highly unlikely that this is in fact the case. Current evidence suggests that hippocampal neural activity makes, at best, a very modest contribution to scalp-recorded potentials (Rugg 1995b), making it improbable that the left parietal old/new effect directly reflects the involvement of the hippocampal formation in episodic retrieval. An alternative possibility, advanced by Wilding and Rugg (1996), is that this effect reflects stimulus-locked changes in cortical activity resulting from the cortico-hippocampal interactions, mentioned in the Introduction, that have been proposed to underlie episodic memory retrieval (McClel- 
land et al. 1995). Wilding and Rugg (1996) further noted that the asymmetric scalp distribution of the effect is strongly suggestive of a generator lateralized to the left hemisphere, as might be expected given the importance of this hemisphere for many aspects of verbal memory (Smith 1989).

The present findings are consistent with Wilding and Rugg's (1996) hypothesis. By this hypothesis, the sensitivity of the left parietal effect to depth of processing arises because the effect represents the hippocampally driven cortical activity that constitutes the neural basis of episodic retrieval (recollection), and recollection is stronger and more probable for deeply studied words than for shallowly studied words. Unfortunately, the present lack of robust methods for localizing the generators of cognitive ERP effects means there is no obvious way of testing this hypothesis directly. The hypothesis does, however, give rise to predictions about the pattern of activation that should be observed in functional neuroimaging studies of the effects of depth of processing on recognition memory: relative to the recognition of shallowly studied words, recognition of deeply studied items should be associated with activation of both the hippocampal formation and cortical regions of the left hemisphere. We now turn to the description of such a study.

\section{Experiment 2}

\section{Method}

The data described here represent some of the findings of a larger study originally reported by Rugg et al. (1997). PET images were obtained while subjects $(n=6)$ performed recognition memory tasks on sequences consisting of words that were either new or which had been studied under deep (sentence generation) or shallow (alphabetic judgement) conditions. Unlike in the ERP study, the two study conditions were blocked, and subjects were administered three study-test blocks for each encoding condition. The study lists consisted of 18 words, and the test lists, which were administered after a filled interval of approximately 2 min, consisted of 18 old and 6 new items (presentation duration $1 \mathrm{~s}$, stimulus onset asynchrony $2.5 \mathrm{~s})$. Subjects signalled their recognition judgement to each item with a verbal response ("old" or "new"). As in the ERP study, item effects were eliminated by rotating words across the different categories (new, deeply studied, shallowly studied).

Measures of regional cerebral blood flow ( $\mathrm{rCBF}$ ) were obtained with ${ }^{15}$ O PET, as described previously (Rugg et al. 1997). Betweencondition differences in $\mathrm{rCBF}$ were identified using the method of statistical parametric mapping (SPM 95, Wellcome Department of Cognitive Neurology, UK). The statistical threshold for all contrasts was $P<0.001$, uncorrected for multiple comparisons.

Results

\section{Behaviour}

Averaged across the three blocks, subjects correctly classified $96 \%$ of the deeply studied old items, along with $94 \%$ of the new items with which they were intermixed. Performance for the shallowly studied words was markedly lower, with $65 \%$ of old words and $83 \%$ of new items

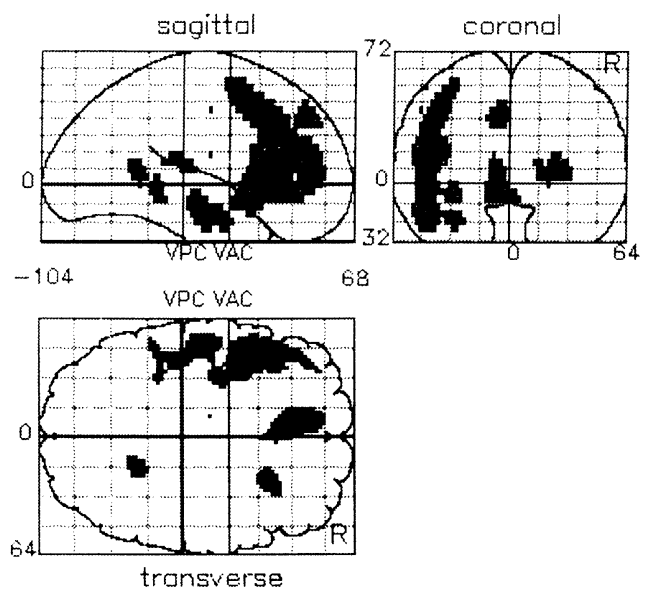

Fig. 3 Experiment 2: maximum intensity projection (threshold $P<0.001$ ) illustrating the cerebral regions in which regional cerebral blood flow was higher during the recognition of deeply studied than shallowly studied words

receiving correct responses. As measured by the discrimination index pHit-pFalse Alarm, recognition was significantly better for the test blocks containing deeply studied words $(t 5=4.41, P<0.01)$.

\section{PET data}

Cerebral regions in which $\mathrm{rCBF}$ was greater during the recognition of words subjected to deep as opposed to shallow encoding are shown in Fig. 3. These regions include the left posterior hippocampus (peak activation at $x, y, z=$ $-32,38,4, Z=3.56$ ), along with an extensive region of left frontal and temporal cortex (peaks at $-46,16,36, Z=4,65$; $-52,18,16, \quad Z=4.19 ; \quad-6,42,36, \quad Z=4.11, \quad-42,-36,27$, $Z=3.64)$ and the left anterior cingulate $(-6,34,-8$, $Z=5.04)$. Additional activations were found in the left thalamus, right caudate/putamen, and right lingual gyrus (Fig. 3).

\section{Discussion}

Two aspects of these results, both predicted from the ERP findings described above, are of particular note. First, in line with the neuropsychological evidence pointing to a role for the hippocampal formation in recollection, this region was more active during the recognition of deeply studied than shallowly studied items. Unlike in two previous reports of hippocampal activation during episodic retrieval (Squire et al 1992; Schacter et al. 1996), both of which employed word stem cued recall rather than recognition memory, the hippocampal activation observed here was strongly left-lateralized, as would be expected on the basis of neuropsychological findings (Smith 1989).

It is worth noting that the majority of functional neuroimaging studies of recognition memory have failed to find evidence for the involvement of hippocampal activation 
in this task (for a review see Fletcher et al. 1997). The predominance of negative findings may reflect the common practice of using new words to provide the baseline against which to assess the effects of successful recognition. New words are most probably subjected to automatic encoding into memory, with concomitant activation of the hippocampus (Nyberg et al. 1996). In the present study the "baseline" consisted of old words which, by virtue of their impoverished encoding, were either unrecollected or recollected only weakly. Such words may engender sufficient familiarity to forestall further memory encoding, and thus provide a more suitable baseline against which to detect hippocampal activation related to successful recollection.

The second notable aspect of the PET findings is that, in addition to the aforementioned hippocampal activation, recognition of deeply studied items was associated with robust and extensive activation of the left frontal and temporal cortex. Activation of these areas has been reported previously in a variety of language tasks (e.g. Price et al. 1996). Given the nature of the deep encoding task (sentence generation), it is possible, therefore, that the activation of these regions during the retrieval of deeply encoded items reflects their role in reinstating the original encoding episode, as envisaged by models of episodic retrieval such as that of McClelland et al. (1995). According to these models, such cortical activity should be under the control of the hippocampal formation. While the present data do not allow this hypothesis to be evaluated, it will be of considerable interest to put it to the test in future studies designed for this purpose.

\section{General discussion}

In both experiments we found clear evidence for neural activity that covaried with recollection, as manipulated by depth of study processing. As discussed below, the findings from the two experiments are highly complementary, although there remains some way to go before data from two such disparate methodologies can be integrated as tightly as one would wish.

The findings from the ERP study with regard to the "left parietal effect" fit well with those from previous work, which also suggest that the effect is a neural correlate of recollection (Allan et al. 1998). Unexpectedly, we found evidence of an earlier, equally robust ERP memory effect that was insensitive to the study manipulation. As already noted, the functional properties of this effect suggest that it reflects a form of non-recollective memory, and it will be of interest to determine whether, in an appropriately designed neuroimaging study of memory retrieval, an analogous dissociation can be found between regions that are sensitive and insensitive to depth of processing at study. It is noteworthy that the two ERP memory effects were dissociated not only by virtue of their differing scalp distributions and functional properties, but also in respect of their time courses; the onset of recollection effect was some $200-250 \mathrm{~ms}$ later than the earlier, depth-insensitive effect. This finding, which exemplifies the power of the ERP method to track patterns of neural activity as they unfold over time, fits well with behavioural evidence indicating that the memory processes supporting familiarity are faster-acting than those responsible for recollection (Hintzman and Curran 1994).

Although at the present time it is not possible to identify with confidence the intracerebral generators of cognitive ERP effects by analysis of their scalp fields, it seems plausible none the less to suppose that a field as asymmetric as that illustrated in Fig. 2 arises from generators strongly lateralized to the left hemisphere. The PET findings - which indicated that the recognition of words subjected to deep encoding gives rise, relative to the recognition of shallowly studied words, to extensive activation of the cortex of the left hemisphere - provide additional support for this proposal.

In neither study was there reliable evidence for the differential activation of right prefrontal cortex in association with the recognition of the deeply studied words. These findings appear at first sight to be in conflict with the results of previous ERP (e.g. Wilding and Rugg 1996) and neuroimaging (e.g. Rugg et al. 1996) studies. The conflict is, however, more apparent than real. In the case of ERPs, right frontally distributed memory effects (presumed to orginate from right prefrontal cortex) for words appear to be task dependent. While there are several reports of such effects in tasks that require more than the mere recognition of study items (e.g. source memory, Wilding and Rugg 1996; associative recognition, Donaldson and Rugg 1998), reports of right frontal memory effects in the kind of task employed here - "yes/no" recognition of words - are rare (Allan and Rugg 1997). With respect to the PET findings, the absence of right frontal activation, a ubiquitous finding in previous studies of episodic retrieval (Fletcher et al. 1997), merely reflects the nature of the contrast that was performed to isolate the effects of depth of processing. This was between two intentional retrieval tasks, in both of which the probability of successsful recognition was well above chance, meaning that in both cases substantial right prefrontal activation would be expected (Fletcher et al. 1997). In keeping with this expectation, activation of this region was observed in both tasks when they were contrasted with a condition in which the requirement was to perform semantic judgements on test words (Rugg et al. 1997).

Finally, it is important to acknowledge the very different preconditions for detecting a signal that must be satisfied with ERP and functional neuroimaging methodologies (Rugg and Coles 1995). Thus, even after setting aside the substantial differences in experimental design between the studies described here, it does not necessarily follow that the two sets of data reflect memory-related activity in equivalent cortical regions, despite the plausibility (and parsimony) of this conclusion in the present case. The development of methods to test the validity of such conclusions directly will mark a major advance in the non-invasive study of brain function. 
Acknowledgements This research was supported by a programme grant from the Wellcome Trust. M.D.R. is supported by a Wellcome Research Leave Fellowship. P.C.F., C.D.F. and R.J.D. are supported by the Wellcome Trust. P.W. was supported by a grant from the Austrian Research Foundation. We thank K. Allan for comments on a earlier version of this paper.

\section{References}

Allan K, Rugg MD (1997) An event-related potential study of explicit memory on tests of word-stem cued recall and recognition memory. Neuropsychologia 35:387-397

Allan K, Wilding EL, Rugg MD (1998) Electrophysiological evidence for dissociable processes contributing to recollection. Acta Psychol 98:231-252

Craik FIM, Lockhart RS (1972) Levels of processing: a framework for memory research. J Verb Learn Verb Behav 11:671-684

Donaldson DI, Rugg MD (1998) Recognition memory for new associations: electrophysiological evidence for the role of recollection. Neuropsychologia (in press)

Fletcher PC, Frith CD, Rugg MD (1997) The functional neuroanatomy of episodic memory. Trends Neurosci 20:213-218

Gardiner JM, Java RI, Richardson-Klavehn A (1996) How level of processing really influences awareness in recognition memory. Can J Exp Psychol 50:114-122

Hintzman DL, Curran T (1994) Retrieval dynamics and recognition and frequency judgments: evidence for separate processes of familiarity and recall. J Mem Lang 33:1-18

McClelland JL, McNaughton BL, O'Reilly RC (1995) Why are there complementary learning systems in the hippocampus and neocortex? Insights from the successes and failures of connectionist models of learning and memory. Psychol Rev 102:419-457

Nyberg L, McIntosh AR, Cabeza R, Habib R, Houle S, Tulving E (1996) General and specific brain-regions involved in encoding and retrieval of events: what, where, and when. Proc Natl Acad Sci USA 93:11280-11285

Paller KA, Kutas M (1992) Brain potentials during retrieval provide neurophysiological support for the distinction between conscious recollection and priming. J Cogn Neurosci 4:375-391

Price CJ, Wise RJS, Warburton EA, Moore, CJ, Howard D, Patterson K, Frackowiak RSJ, Friston KJ (1996) Hearing and saying: the functional neuroanatomy of auditory word-processing. Brain 119:919-931

Rugg MD (1995a) Memory and consciousness: a selective review of issues and data. Neuropsychologia 33:1131-1142

Rugg MD (1995b) Cognitive event-related potentials: intracranial and lesion studies. In: Baron JC, Grafman J (eds) Handbook of neuropsychology, vol 10. Elsevier, Amsterdam, pp 165-186

Rugg MD, Coles MGH (1995) The ERP and cognitive psychology: conceptual issues. In: Rugg MD, Coles MGH (eds) Electrophysiology of mind: event-related brain potentials and cognition. Oxford University Press, Oxford, pp 27-39

Rugg MD, Fletcher PC, Frith CD, Frackowiak RSJ, Dolan RJ (1996) Differential activation of the prefrontal cortex in successful and unsuccessful memory retrieval. Brain 119:2073

Rugg MD, Fletcher PC, Frith CD, Frackowiak RSJ, Dolan RJ (1997) Brain regions supporting intentional and incidental memory: a PET study. Neuroreport 8:1283-1287

Rugg MD, Mark RE, Walla P, Schloerscheidt AM, Birch CS, Allan K (1998) Dissociation of the neural correlates of implicit and explicit memory. Nature 392:595-598

Schacter DL, Alpert NM, Savage CR, Rauch SL, Albert MS (1996) Conscious recollection and the human hippocampal formation: evidence from positron emission tomography. Proc Natl Acad Sci USA 93:321-325

Smith ME (1993) Neurophysiological manifestations of recollective experience during recognition memory judgements. J Cogn Neurosci 5:1-13

Smith ML (1989) Memory disorders associated with temporal lobe lesions. In: Boller JC, Grafman J (eds) Handbook of neuropsychology, vol 3. Elsevier, Amsterdam, pp 91-106

Squire LR, Ojemann JG, Miezin FM, Petersen SE, Videen TO, Raichle ME (1992) Activation of the hippocampus in normal humans: a functional anatomical study of memory. Proc Natl Acad Sci USA 89:1837-1841

Squire LR, Knowlton B, Musen G (1993) The structure and organization of memory. Annu Rev Psychol 44:453-495

Toth JP (1996) Conceptual automaticity in recognition memory: levels of processing effects on familiarity. Can J Exp Psychol 50: $123-138$

Wilding EL, Rugg MD (1996) An event-related potential study of recognition memory with and without retrieval of source. Brain 119:889-906 